\title{
Revista Colombiana de

\section{Programa de prevención y rehabilitación cardiaca: herramienta útil y necesaria en el tratamiento del ataque cerebrovascular}

\section{Prevention and Cardiac Rehabilitation Program: useful and necessary tool in the treatment after stroke}

\author{
Mónica Rincón ${ }^{\mathrm{a}, \mathrm{b}}$ \\ a Servicio de Rehabilitación, Fundación Cardioinfantil-Instituto de Cardiología, Bogotá, Colombia \\ b Universidad del Rosario y Universidad de La Sabana, Bogotá, Colombia
}

Recibido el 2 de marzo de 2015; aceptado el 3 de marzo de 2015

El Observatorio Nacional de Salud del Instituto Nacional de Salud de Colombia, en su Boletín N. ${ }^{\circ} 1$ de diciembre 9 de 2013, establece que las enfermedades cardiovasculares representan la primera causa de muerte en el país. En el periodo 1998-2011 se registraron 628.630 muertes por enfermedades cardiovasculares, que corresponden al 23,5\% del total de las muertes en Colombia. De las defunciones atribuidas al grupo de enfermedad cardiovascular seleccionado, un $56,3 \%$ obedece a enfermedad cardiaca isquémica, 30,6\% a enfermedad cerebrovascular, 12,4\% a enfermedad hipertensiva y $0,5 \%$ a enfermedad cardiaca reumática crónica ${ }^{1}$. A pesar de estas cifras y del conocido impacto social y económico que generan estas enfermedades, no se presta una atención adecuada a las enfermedades cerebrovasculares, una preocupación que no debe dejarse de lado y que implica una intervención acertada desde diferentes especialidades médicas, así como también una implementación de estrategias que mejoren la carga generada por esta.

Véase contenido relacionado en DOI:

http://dx.doi.org/10.1016/j.rccar.2015.03.002

Correo electrónico: mrinron@hotmail.com
Un artículo reciente publicado en The Lancet $^{2}$, presenta el estudio de carga regional y global del ataque cerebrovascular (ACV) durante 1990 a 2010, en el que se muestra cómo su incidencia aumentó en un $12 \%$ en países de medio y bajo ingreso, mientras que en los de alto ingreso se redujo en el mismo porcentaje. Así mismo, el número absoluto de personas con un primer episodio de ACV, las muertes a causa de este y los años de vida perdidos ajustados en función de la discapacidad (AVAD), fueron más altos y han venido incrementándose significativamente desde 1990, en países de medio y bajo ingreso. De igual forma, los autores de este estudio muestran cómo el número de AVAD perdidos en personas menores de 75 años en países de medio y bajo ingreso, excedió en más de cinco veces a los de países de alto ingreso y dos veces en las personas mayores de 75 años. Es así como los estudios ${ }^{3}$ han establecido que el ACV es la causa líder de discapacidad neurológica en adultos, y que el pobre nivel de acondicionamiento físico, incluida una baja capacidad funcional, son los factores que limitan a los pacientes para realizar sus actividades de la vida diaria e impactan en el riesgo de sufrir un nuevo episodio e incrementar la mortalidad por esta causa. Los pacientes en un período posterior a un ACV mayor de seis meses, cuentan con un 55 
a 75\% de la capacidad funcional esperada para una persona saludable del mismo género, edad y condición física. Esta disminución de su capacidad funcional acarrea efectos negativos en la movilidad y en la resistencia a la fatiga, y empeora aún más su desempeño funcional e independencia, llevándolos a una mayor restricción de su participación en la comunidad ${ }^{4}$.

De igual manera, se sabe que cerca del $75 \%$ de los pacientes con ACV tiene enfermedad cardiaca y comparten los mismos factores de riesgo. Por ejemplo, se conoce que la diabetes mellitus es un fuerte predictor independiente de esta patología en el primer año después de un ataque isquémico transitorio, con una tasa de recurrencia de ACV sustancialmente mayor ${ }^{5}$.

De otro lado, la inactividad física y el aumento del perímetro abdominal son factores independientes para un ACV primario, y respecto al tabaquismo el cual tiene una relación causal con la arterioesclerosis, explica por qué es un factor de riesgo mayor e independiente para un nuevo evento cerebrovascular ${ }^{6}$. Además, la enfermedad cardiovascular es el mayor factor que limita los resultados exitosos en la rehabilitación después de un ACV.

Lo anterior demuestra la existencia de una estrecha relación entre enfermedad coronaria y un evento cerebrovascular en cuanto a etiología, enfermedades asociadas y factores de riesgo, que a su vez sugiere que las estrategias que se han utilizado con éxito para el tratamiento de la enfermedad coronaria pueden ser útiles para el abordaje de esta patología.

De ahí el interés de llamar la atención al grupo de salud que se encarga del cuidado de los pacientes con enfermedad cardiovascular, en cuanto a iniciar la implementación de los programas de Prevención y Rehabilitación Cardiaca para los pacientes con ACV, tradicionalmente enfocados en la educación, el ejercicio y la intervención de los factores de riesgo cardiovascular.

El entrenamiento cardiovascular y respiratorio que ofrecen estos programas disminuye la fatiga, la incidencia de caídas y fracturas, al tiempo que brinda una mejor compensación del costo energético de la marcha hemiparética ${ }^{7}$. De igual manera, reduce la discapacidad por su impacto en la movilidad, el balance corporal y el equilibrio, entre otros. Los pacientes con este tipo de entrenamiento mejoran su independencia funcional, la percepción de su calidad de vida y el estado anímico ${ }^{8}$.

Diferentes revisiones sistemáticas muestran una reducción significativa de factores de riesgo como presión arterial, obesidad, diabetes mellitus y mortalidad por enfermedad coronaria, además de una mejoría notable de la depresión en aquellos pacientes que asisten a los programas de rehabilitación cardiaca?.

Para el grupo interdisciplinario de salud que se encarga del tratamiento de estos pacientes, es relevante lograr la integración de los mismos a su vida diaria. Por eso, diferentes estudios han demostrado cómo los pacientes que ingresan en programas de prevención y rehabilitación cardiaca presentan una mejoría clínica significativa en la participación en las actividades de la vida diaria percibida a través de la escala de impacto del ACV. Esta participación, que se traduce en la integración a la comunidad, es el predictor principal de la recuperación global de una persona con discapacidad.
La American Heart Association propone un programa que incluya entrenamiento cardiovascular y educación para mejorar los factores de riesgo cardiovascular y recomienda su utilización para sobrevivientes de ACV como parte de un programa de reducción del riesgo cardiovascular y de un nuevo evento cerebrovascular.

Diferentes modelos cuantitativos han concluido que al menos el $80 \%$ de los eventos vasculares recurrentes después de un ACV o ataque isquémico transitorio inicial, puede prevenirse a través de una estrategia multifactorial ${ }^{10}$. Es así como los programas de prevención y rehabilitación cardiaca ofrecen una oportunidad para reducir el riesgo cardiovascular, la mortalidad y la recurrencia de un ACV y del mismo modo han demostrado mejorar la percepción de la calidad de vida debido a sus componentes de ejercicio y a la educación que se imparte ${ }^{11}$.

En los países de alto ingreso, los servicios de salud competentes y las estrategias para prevención y cuidado del ACV (modificación de factores de riesgo cardiovascular, unidades de tratamiento de ACV agudo) son los mejores indicadores para reducir la incidencia de eventos cerebrovasculares, mortalidad y pérdida de AVAD, contrario a lo que sucede en los países de medio y bajo ingreso como Colombia.

La reducción en la prevalencia, la modificación y el control de los factores de riesgo, y la accesibilidad a los servicios de salud, incluidas las unidades de cuidado agudo, rehabilitación y prevención secundaria, son los factores que marcan la diferencia en la carga que genera esta enfermedad en los diversos países.

Existe una preocupante tendencia al aumento de la incidencia de ACV en adultos jóvenes debido al incremento mundial de diabetes y a la prevalencia creciente de otros factores de riesgo cardiovascular en todos los grupos poblacionales, sobre todo en países de bajo y medio ingreso. Es probable que el cambio en la carga del ACV en las poblaciones más jóvenes, continúe en todo el mundo a menos que se establezcan estrategias preventivas, dentro de las cuales no pueden excluirse los programas de prevención y rehabilitación cardiaca.

Por esta razón, la propuesta actual es considerar el modelo de rehabilitación cardiaca como una estrategia de prevención secundaria en la atención del ACV, pues ha demostrado de manera significativa un impacto positivo en la reducción de la morbimortalidad, al lograr uno de los objetivos más importantes en el tratamiento de estos pacientes: la mejoría notoria en la percepción de su calidad de vida ${ }^{12}$

Esto podrá alcanzarse al ser conscientes de la existencia de esta estrategia y su implementación. Considero, por tanto, que la clave del desafío está en crear grupos interdisciplinarios de salud, bien entrenados, que puedan tratar personas con discapacidad física, comunicativa, sensorial, cognoscitiva y con afectación cardiovascular ${ }^{13}$.

El costo que genera la discapacidad por ACV tiene implicaciones a diferentes niveles; no solo se trata del costo económico, sino del emocional, familiar y social, de ahí que nuestro deber sea buscar y hacer uso de las herramientas disponibles para lograr que las personas con discapacidad vuelvan a incorporarse en las mejores condiciones posibles, a todas las actividades en las que participaban antes de sufrir el evento. 
Porque nuestros pacientes lo merecen, y en nuestro medio contamos con esta intervención, es necesario que todos los profesionales del equipo de salud tengan presente dentro del tratamiento para estos pacientes, la remisión a los programas de prevención y rehabilitación cardiaca. No dejemos de lado esta importante oportunidad.

\section{Bibliografía}

1. Observatorio Nacional de Salud (ONS). Instituto Nacional de Salud de Colombia (INS). Boletín N. ${ }^{\circ}$ 1; diciembre 9 de 2013. Bogotá: Ministerio de Salud y Protección Social.

2. Feigin VL, Forouzanfar MH, Krishnamurthi R, Mensah GA, Connor M, Bennett DA, et al. Global burden of Diseases, Injuries, and Risk Factors Study 2010 (GBD 2010) and the GBD Stroke Experts Group. Global and regional burden of stroke during 1990-2010: findings from the Global Burden of Disease Study 2010. Lancet. 2014;383:245-55.

3. Booth JN, Levitan EB, Brown TM, Farkouh ME, Safford MM, Muntner P. Effect of sustaining lifestyle modifications (nonsmoking, weight reduction, physical activity, and mediterranean diet) after healing of myocardial infarction, percutaneous intervention, or coronary bypass (from the REasons for Geographic and Racial Differences in Stroke Study). Am J Cardiol. 2014;113(12):1933-40.

4. Marzolini S, Tang A, Mcllroy W, Oh PI, Brooks D. Outcomes in people after stroke attending an adapted cardiac rehabilitation exercise program: does time from stroke make a difference? J Stroke Cerebrovasc Dis. 2014;23(6): 1648-56.
5. Tang A, Marzolini S, Oh P, Mcllroy WE, Brooks D. Feasibility and effects of adapted cardiacrehabilitation after stroke: a prospective trial. BMC Neurol. 2010;10:40.

6. MacKay-Lyons M, Gubitz G, Giacomantonio N, Wightman H, Marsters D, Thompson K, et al. Program of rehabilitative exercise and education to avert vascular events after non-disabling stroke or transient ischemic attack (PREVENT Trial): a multicentred, randomised controlled trial. BMC Neurol. 2010;10:122.

7. Prior PL, Hachinski V, Unsworth K, Chan R, Mytka S, O'Callaghan C, et al. Comprehensive cardiac rehabilitation for secondary prevention after transient ischemic attack or mild stroke: I: feasibility and risk factors. Stroke. 2011;42(11):3207-13.

8. Saunders DH, Sanderson M, Brazzelli M, Greig CA, Mead GE. Physical fitness training for stroke patients. Cochrane Database Syst Rev. 2013, http://dx.doi.org/10.1002/14651858, 10CD003316.

9. Stoller O, de Bruin ED, Knols RH, Hunt KJ. Effects of cardiovascular exercise early after stroke: systematic review and meta-analysis. BMC Neurol. 2012;12:45.

10. Lindsay P, Furie KL, Davis SM, Donnan GA, Norrving B. World stroke organization global stroke services guidelines and action plan. Int J Stroke. 2014;9 Suppl A100:4-13, http://dx.doi.org/10.1111/ijs.12371. Epub 2014 Sep 23.

11. Rimmer CM. Effects of exercise on quality of life in stroke survivors: a meta-analysis. Stroke. 2011;42:832-7.

12. Wang Z, Wang L, Fan H, Jiang W, Wang S, Gu Z, Wang T. Adapted low intensity ergometer aerobic training for early and severely impaired stroke survivors: a pilot randomized controlled trial to explore its feasibility and efficacy. J PhysTher Sci. 2014;26(9):1449-54.

13. Tang A, Closson V, Marzolini S, Oh P, Mcllroy W, Brooks D. Cardiac rehabilitation after stroke-need and opportunity. J Cardiopulm Rehabil Prev. 2009;29(2):97-104. 